\title{
A GAME THEORETICAL ALGORITHM FOR JOINT POWER AND TOPOLOGY CONTROL IN DISTRIBUTED WSN
}

\author{
Pau Closas, Alba Pagès-Zamora and Juan A. Fernández-Rubio \\ Universitat Politècnica de Catalunya (UPC), Dept. of Signal Theory and Communications \\ Campus Nord, 08034 Barcelona, Spain. e-mail: \{closas,alba,juan\} @ gps.tsc.upc.edu
}

\begin{abstract}
In this paper, the issue of network topology control in wireless networks using a fully distributed algorithm is considered. Whereas the proposed distributed algorithm is designed applying game theory concepts to design a non-cooperative game, network connectivity is guaranteed based on asymptotic results of network connectivity. Simulations show that for a relatively low node density, the probability that the proposed algorithm leads to a connected network is close to one.
\end{abstract}

Index Terms- Wireless Sensor Networks, Distributed algorithms, Game theory.

\section{INTRODUCTION}

Wireless sensor networks (WSNs) have received much attention in recent years as a technology useful to estimate physical parameters of a given area in order to, for instance, monitor processes or detect and prevent environmental disasters. Some WSN applications, e.g. forest fires, may require a large amount of nodes. The feasibility of these networks thus relies on the development of cheap nodes with low energy consumption and limited processing capabilities. In these networks, the use of distributed processes based on the exchange of local information among the nodes only is of great interest.

One important issue in WSN is the topology control problem since it plays a fundamental role in the behavior of the network. A connected network (or group of nodes if the network is organized in clusters) is usually required in order to ensure the delivery of packets to a fusion center in a centralized WSN, or in order to reach a consensus in the estimation or detection of events in decentralized WSNs. Since connectivity may vary with time due to malfunctioning nodes or node mobility, the network should be able to dynamically adjust the topology, preferably, in a self-organized way. Several topology control algorithms can be found in the literature [1], they are either centralized algorithms that require global network information or distributed algorithms with a constraint on the

This work has been partially funded by the European Commission (033914-WINSOC), the Spanish Ministry of Education and Science and FEDER funds (TEC2006-06481 and CONSOLIDER CSD2008-00010 COMONSENS) and the Catalan Government (2005SGR-00639). minimum number of neighbors each node should have to ensure certain topology properties.

In this paper we address the problem of network topology control and propose a distributed algorithm to adjust the transmission power of each node formulated as a noncooperative game where nodes exchange information only with their neighbors. Game theory is a collection of models and analytic tools useful to study interactive decision processes $[2,3]$. Therefore, it provides a framework to solve in a distributive fashion the problem of adjusting the transmission power of the nodes in a WSN guaranteeing connectivity and with an energy-efficient solution. In [1] the authors present examples of topology control games and a distributed algorithm that guarantees convergence to a connected network. However, this algorithm requires global information flowing through the network to check at each iteration the connectivity or not of the network. Our contribution relaxes this assumption and proposes a fully distributed algorithm based on local information only.

The paper is organized as follows. In Section 2 we introduce some general concepts of game theory. In Section 3 we present the game proposed to adjust the node transmission power and a procedure to locally estimate the connectivity of the network. Section 4 shows the simulation results and Section 5 concludes the paper.

\section{GAME THEORY BASICS}

We limit our discussion to non-cooperative models that address the interaction among individual rational decision makers. Such models are called games and the rational decision makers are referred to as players.

A strategic non-cooperative game $\Gamma(\Omega, A, u)$ has three main components:

- $\Omega$ is the players set, s.t. $\Omega=1, \ldots, N$ with $N$ the number of players in the game.

- $A$ is the set of strategies and $\mathbf{a}=\left[a_{1}, \ldots, a_{N}\right]^{T} \in A=$ $\times_{i=1}^{N} A_{i} . \quad a_{i} \in A_{i}$ represents the strategy of the $i$-th player over the set of its possible strategies. Similarly, $\mathbf{a}_{-i} \in A_{-i}=\times_{j \neq i}^{N} A_{j}$ represents the strategies of the rest of $N-1$ players. 
- $u_{i}: A \rightarrow \mathbb{R}^{N}$ is the utility function of the $i$-th player. The utility function (or payoff) quantifies the preferences of each user to a given strategy. Then, $\mathbf{u}=$ $\left[u_{1}, \ldots, u_{N}\right]^{T}$ is the vector of all $N$ utility functions.

Then, a non-cooperative game is an iterative procedure where, at each iteration, players chose the strategy that maximizes their utility function. The Nash equilibrium (NE) is a stable solution of the game in which no player may improve its utility function by unilaterally deviating from it.

Definition 2.1 (Nash Equilibrium) An strategy profile $\mathbf{a}^{*}$ is a Nash equilibrium if, $\forall i \in \Omega$ and $\forall a_{i} \in A$,

$$
u_{i}\left(\mathbf{a}^{*}\right) \geq u_{i}\left(a_{i}, \mathbf{a}_{-i}^{*}\right)
$$

In general, games may have a large number of NE or may not have any. Thus, it is of interest to design the utility function in a way such that the game has at least one equilibrium point. It is proved in [4] that when the utility functions used by the players are concave, the existence and uniqueness of a NE is proved. However, the utility function may be designed according to a criteria which could eventually yield to non-convex functions. In those cases, there is another way for deriving sufficient conditions for existence and uniqueness of the NE in a game based on the so-called potential games, [5]. This type of games is given when the incentive of all players to change their strategy can be expressed by a global utility function $V$. We refer to an exact potential game (EPG) when the game admits an exact potential function, i.e., a player-independent real valued function that measures the marginal payoff when any player deviates unilaterally (see [5] for details). An ordinal potential game (OPG) is another type of potential games which require having an ordinal potential function that has the same directional behavior as the individual payoff function, when that individual unilaterally deviates. More rigorous definitions follow.

Definition 2.2 (EPG) A strategic game $\Gamma(\Omega, A, u)$ is an exact potential game if there exist an exact potential function $V: A \rightarrow \mathbb{R}$ s.t. $\forall i \in \Omega, \forall \mathbf{a}_{-i} \in A_{-i}$ and $\forall a_{i}, b_{i} \in A_{i}$

$$
V\left(a_{i}, \mathbf{a}_{-i}\right)-V\left(b_{i}, \mathbf{a}_{-i}\right)=u_{i}\left(a_{i}, \mathbf{a}_{-i}\right)-u_{i}\left(b_{i}, \mathbf{a}_{-i}\right)
$$

Definition 2.3 (OPG) A strategic game $\Gamma(\Omega, A, u)$ is an ordinal potential game if there exist an ordinal potential function $V: A \rightarrow \mathbb{R}$ s.t. $\forall i \in \Omega, \forall \mathbf{a}_{-i} \in A_{-i}$ and $\forall a_{i}, b_{i} \in A_{i}$

$V\left(a_{i}, \mathbf{a}_{-i}\right)-V\left(b_{i}, \mathbf{a}_{-i}\right)>0 \Leftrightarrow u_{i}\left(a_{i}, \mathbf{a}_{-i}\right)-u_{i}\left(b_{i}, \mathbf{a}_{-i}\right)>0$

As stated in the following Lemma (see [5] for the proof), the game defined by the utility function $V$ presents the same NE points as the original game whenever it is an OPG game. Lemma 2.4 also holds for an EPG game since it is a particular case of an OPG game.

Lemma 2.4 Let $\Gamma(\Omega, A, u)$ be an $O P G$ and $V$ the corresponding ordinal potential function. If $\mathbf{a} \in A$ maximizes $V$, then it is a NE.

\section{GAME THEORETICAL APPROACH TO DISTRIBUTED POWER AND TOPOLOGY CONTROL}

The aim of this section is to develop a fully distributed algorithm to adjust the transmission power of each node in a WSN so that the network becomes connected with an energy-efficient solution. The algorithm is formulated as a non-cooperative game where nodes exchange information only with their neighbors.

\subsection{A Game Theoretical Algorithm}

For the problem at hand, the players are the nodes and the game is that of finding a NE such that each node is transmitting at a minimal power while maintaining the network connected. Thus, the set of strategies that the $i$-th node can chose are the set of its possible discrete power levels $P_{i}$. Consequently, we can define the instantaneous vector of power levels as $\mathbf{p}=\left[p_{1}, \ldots, p_{N}\right]^{T} \in P=\times_{i=1}^{N} P_{i}$ as the vector containing the power of each node. We also assume that, at the beginning of the game, the nodes transmit with their maximum power level to gather neighbors information and the network is connected.

In order to achieve the NE in the strategic non-cooperative game $\Gamma(\Omega, P, u)$, nodes iteratively decide its power transmission by maximizing its utility function,

$$
\hat{p}_{i}=\arg \max _{p_{i} \in P_{i}}\left\{u_{i}\left(p_{i}, \hat{\mathbf{p}}_{-i}\right)\right\}
$$

After each iteration, a node power level change affects the overall topology of the network which is taken into account by the other nodes when optimizing their utility function.

As mentioned in Section 2, the iterative best response algorithm in (4) yields to a NE or not depending on the design of the utility function. Therefore, we aim at designing a utility function with a NE that preserves connectivity but with a more energy efficient solution than the initial topology with all nodes transmitting at maximum power. Following the result in [1], the utility function of Proposition 3.1 is considered.

Proposition 3.1 The game $\Gamma(\Omega, P, u)$ where the individual utilities are given by

$$
u_{i}\left(p_{i}, \mathbf{p}_{-i}\right)=\left\{\begin{array}{cc}
p_{i, \max }-p_{i} & \text { if network is connected } \\
-p_{i} & \text { otherwise }
\end{array}\right.
$$

is an EPG and the exact potential function is

$V\left(p_{i}, \mathbf{p}_{-i}\right)=\left\{\begin{array}{cc}p_{i, \max }-\sum_{i \in \Omega} p_{i} & \text { if network is connected } \\ -\sum_{i \in \Omega} p_{i} & \text { otherwise. }\end{array}\right.$

Proof: We prove it by applying the concept of EPG in Definition 2.2. Consider $q_{i}, p_{i} \in P$ s.t. $q_{i}<p_{i}$, then we have 
that

$$
\Delta u_{i}=u_{i}\left(p_{i}, \mathbf{p}_{-i}\right)-u_{i}\left(q_{i}, \mathbf{p}_{-i}\right)=q_{i}-p_{i}
$$

whether the network is connected or not. Similarly, we construct the potential variational as

$$
\begin{aligned}
\Delta V & =V\left(p_{i}, \mathbf{p}_{-i}\right)-V\left(q_{i}, \mathbf{p}_{-i}\right) \\
& =-\left(p_{i}+\sum_{j \in \Omega ; j \neq i} p_{j}\right)+\left(q_{i}+\sum_{j \in \Omega ; j \neq i} p_{j}\right)
\end{aligned}
$$

Thus, $\Delta u_{i} \equiv \Delta V \Rightarrow V$ is an exact potential function and the game $\Gamma(\Omega, P, u)$ is an EPG.

The designed game falls in the category of EPG games and, as stated in Lemma 2.4, finding the NE point of (5) is equivalent to maximize the potential function in (6). If we examine (5) and (6), we may see that the value of $p_{i}$ that maximizes both the utility function and $\mathrm{V}$ is the minimum transmission power for what the network is connected, if any.

For the sake of clarity, a pseudocode of the procedure is shown in Algorithm 1. That is the sequence of iterations that a node $i$ executes before deciding its own power level, $p_{i}$. Notice that each node runs the best response algorithm in (4) $N_{i t e r}$ times in parallel and not necessarily synchronized. It is important to remark that we assume each node knows the transmission power level used by its neighbors. This information can be easily included in the packets exchanged between nodes if there is a discrete set of power levels.

At the light of the proposed utility function, the main concern is to determine whether the network is connected or not. This point was not addressed in [1] which was mentioned as future work. However, this point is challenging if one aims at designing a fully distributed algorithm that uses local information only. In Section 3.2 the issue of determining network connectivity with only local information is addressed.

\subsection{Distributed Connectivity decision}

In order to check the connectivity of a network we have to resort to graph theory principles. This theory describes the communication flow among the nodes of a network by an undirected graph $\mathscr{G}=(\mathscr{V}, \mathscr{E})$ where $\mathscr{V}=\{1, \cdots, N\}$ is the set of vertices (nodes) and $\mathscr{E}$ is the set of edges (links) [6]. The edge $e_{i j}$ represents a bidirectional communication link between a pair of distinct nodes $i$ and $j$. The set of neighbors of node $i$ is defined as $\mathcal{N}_{i}=\left\{j \in \mathscr{V}: e_{i j} \in \mathscr{E}\right\}$ for all $i, j=\{1, \cdots, N\}$, and represents the set of indexes of the nodes sending information to node $i$. A path in a graph $\mathscr{G}$ is a sequence of alternating nodes and edges that starts and ends at different nodes, such that each edge is incident to its predecessor and successor node. The graph is connected if there exists a path between any two nodes, and the connectivity (or

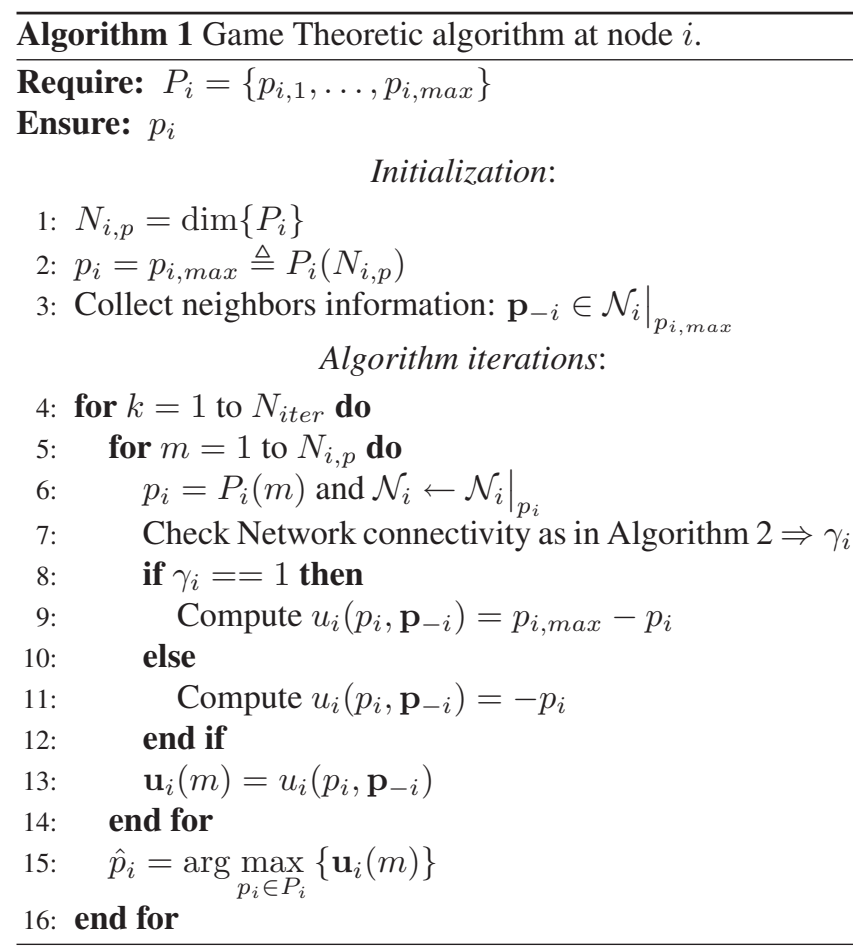

topology) of the graph is described by the $N \times N$ Laplacian matrix $\mathbf{L}$, with entries

$$
[\mathbf{L}]_{i j}=\left\{\begin{array}{rl}
d_{i}^{(\text {out })} & i=j \\
-1 & i \neq j \& e_{i j} \in \mathscr{E} \\
0 & \text { otherwise }
\end{array}\right.
$$

where $d_{i}^{\text {(out) }}$ stands for the outdegree of node $i$, and corresponds in our case to $\mathcal{N}_{i}$. If the set $\lambda_{1} \leq \lambda_{2} \leq \cdots \leq \lambda_{N}$ denote the eigenvalues of $\mathbf{L}$, by definition we have $\lambda_{1}=0$. If the graph is connected, from spectral graph theory results we have that the algebraic multiplicity of $\lambda_{1}$ is equal to one and $\mathbf{L}$ is an irreducible matrix [7].

Therefore, global information of the network is needed to check its connectivity since the computation of the second smallest eigenvalue of the Laplacian is required. However, we are interested in a procedure to check connectivity but based on local information only. In [8] the authors have obtained a lower bound on the communication radius $r(n)$ in order to ensure connectivity with a high probability. Specifically, it is shown that if $n$ nodes are placed in a disc of unit area in $\mathbb{R}^{2}$ and each node transmits at a power level so as to cover an area of $\pi r^{2}(n)=(\log (n)+c(n)) / n$, then the resulting network is asymptotically connected with probability one if and only if $c(n) \rightarrow+\infty$. A common choice for $c(n)$ is $c(n)=K \log (n)$ with $K>0$. The radius $r^{*}$ computed as

$$
r^{*}=\sqrt{\frac{\log (n)}{n}}
$$

can be used as an asymptotical threshold so that if $r(n)$ decreases faster than $r^{*}$, then the probability that the network is 
connected tends to zero as $n$ increases. Conversely, if $r(n)$ decreases slower than $r^{*}$, then the probability that the network is connected tends to one. The methodology proposed to check connectivity is detailed in Algorithm 2. For each power level, $p_{i}$, the node estimates the node density as the relation between the number of neighbors transmitting with a power less or equal to $p_{i}$ and the coverage area. Then, the threshold $\hat{r}_{i}^{*}$ is estimated with this node density. The decision on the connectivity is taken comparing the actual coverage radio $r_{i}$ with the threshold. Since this procedure is based on an asymptotic threshold function, its performance will improve as the node density increases.

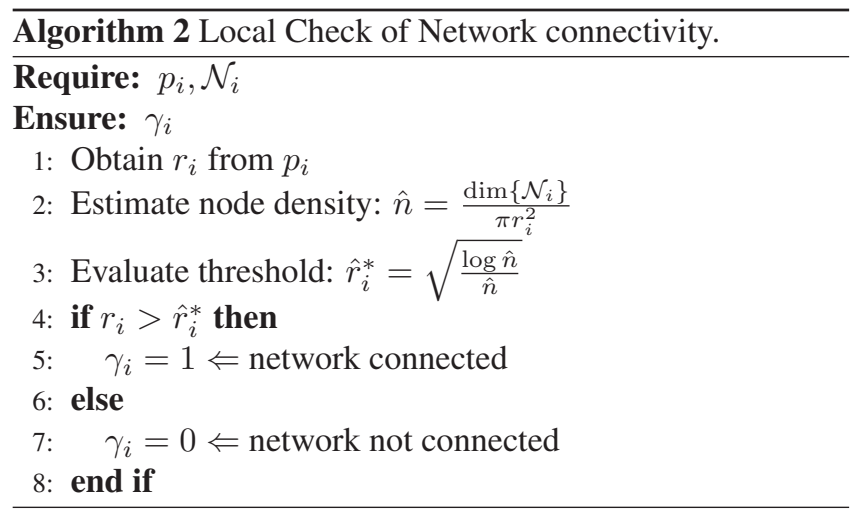

\section{SIMULATION RESULTS}

The proposed algorithm has been simulated and validated by computer simulation. The WSN is generated by randomly placing nodes in a $10 \times 10$ meters square. We consider the same set of discrete power levels for each node. Since the decision on network topology is based on asymptotical assumptions, i.e. when $n \rightarrow \infty$, the algorithm has been tested in order to evaluate its validity. Figure 1 shows the probability of having a connected graph after running the algorithm versus node density deployed in the field area. Results show that it is required a minimum density of nodes to guarantee a certain probability of obtaining a connected network.

\section{CONCLUSIONS}

This paper studies the problem of power and topology control in a distributed WSN using game theory tools. The novelty of the work is that network connectivity is achieved with a fully distributed algorithm based on a non-cooperative game as the node density increases. The proposed algorithm is based on a non-cooperative game and asymptotic results for network connectivity. The algorithm is validated through computer simulation results, were the asymptotic behavior as the number of nodes increase becomes apparent.

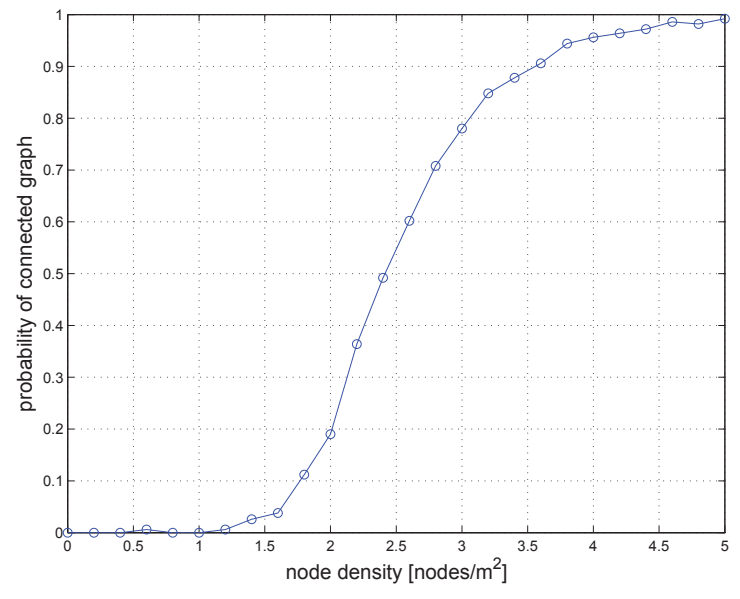

Fig. 1. Probability of having a connected graph as a function of node density.

\section{REFERENCES}

[1] Ramakant S. Komali and Allen B. MacKenzie, "Effect of selfish node behaviour on efficient topology design," IEEE Trans. on Mobile Computing, vol. 7, no. 6, June 2008.

[2] D. Fudenberg and J. Tirole, Game Theory, Cambridge: The MIT press, 1991.

[3] V. Srivastava et al., "Using game theory to analyze wireless ad-hoc networks," IEEE Communications Surveys and Tutorials, vol. 7, no. 4, pp. 46-56, 2005.

[4] J. B. Rosen, "Existence and uniqueness of equilibrium points for concave n-person games," Econometrica, vol. 33, no. 3, pp. 520-534, 1965.

[5] D. Monderer and L. S. Shapley, "Potential games," Games and Economics Behaviour, vol. 14, no. 14, pp. 124-143, 1996.

[6] C. Godsil and G. Royle, Algebraic graph theory, vol. 207, Graduate Texts in Mathematics. Berlin, Germany: Springer-Verlag, 2001.

[7] R.A. Horn and C.R. Johnson, Matrix analysis, Cambridge University Press, 2006.

[8] P. Gupta and P .R. Kumar, Stochastic Analysis, Control, Optimization and Applications: A Volume in Honor of W.H. Fleming, chapter 33: Critical power for asymptotic connectivity in wireless networks, pp. 547-566, Birkhauser, Boston, MA, 1998. 